\title{
Combination of oncolytic adenovirus and endostatin inhibits human retinoblastoma in an in vivo mouse model
}

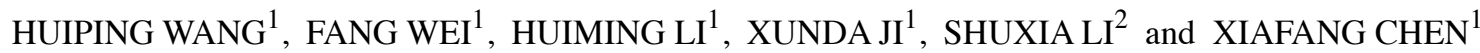 \\ ${ }^{1}$ Experimental Research Center, The First People's Hospital, Shanghai Jiaotong University, Shanghai 201620; \\ ${ }^{2}$ Department of Pathology, Shanghai First Maternity and Infant Hospital, Tongji University \\ School of Medicine, Shanghai 200040, P.R. China
}

Received March 22, 2012; Accepted June 6, 2012

DOI: $10.3892 / \mathrm{ijmm} .2012 .1197$

\begin{abstract}
There is a critical need for new paradigms in retinoblastoma (RB) treatment that would more efficiently inhibit tumor growth while sparing the vision of patients. Oncolytic adenoviruses with the ability to selectively replicate and kill tumor cells are a promising strategy for cancer gene therapy. Exploration of a novel targeting strategy for RB utilizing combined oncolytic adenovirus and anti-angiogenesis therapy was applied over the course of the current study with positive results. The oncolytic adenoviruses Ad-E2F1 p-E1A and Ad-TERT p-E1 were constructed. The E1 region was regulated by the E2F-1 promoter or the human telomerase reverse transcriptase (hTERT) promoter, respectively. Effects on both replication and promotion of enhanced green fluorescent protein (EGFP) expression were observed in the replicationdefective adenovirus Ad-EGFP in diverse cancer cell lines, HXO-RB44, Y79, Hep3B, NCIH460, MCF-7 and HLF. The cancer cell death induced by these agents was also explored. The in situ RB model demonstrated that mice with tumors treated with the oncolytic adenovirus and replication-defective adenovirus Ad-endostatin exhibited notable cancer cell death. This anticancer effect was further examined by stereo microscope, and the survival rate of experimental mice was determined. Both Ad-E2F1 p-E1A and Ad-TERT p-E1 replicated specifically in cancer cells in vitro and promoted EGFP expression in Ad-EGFP, although Ad-E2F1 p-E1A demonstrated superior EGFP promotion activity than Ad-TERT p-E1. In Hep3B, NCIH460 and MCF-7 cells, the number of Ad-TERT p-E1 copies was observed to exceed of the number of Ad-E2F1 p-E1A copies by a minimum of 10-fold. Furthermore, Ad-TERT p-E1 demonstrated significantly superior oncolytic
\end{abstract}

Correspondence to: Dr Fang Wei, Experimental Research Center, The First People's Hospital, Shanghai Jiaotong University, 650 New Songjiang Road, Shanghai 201620, P.R. China

E-mail: weifang73@126.com

Key words: human retinoblastoma, oncolytic adenovirus, replicationdefective adenovirus, endostatin, E2F1, E1A, E1B effects in the RB mouse model, and Ad-endostatin effectively suppressed tumor growth and extended the overall lifespan of subjects; however, the Ad-E2F1 p-E1A was clearly less effective in attaining these goals. Most notably, the antitumor effect and survival rate of subjects in the combined Ad-TERT p-E1 + Ad-endostatin group were higher than those treated with either single Ad-TERT p-E1 ( $\mathrm{p}=0.097, \mathrm{p}=0.022$, respectively) or Ad-endostatin ( $\mathrm{p}=0.037, \mathrm{p}=0.006$, respectively). In conclusion, application of transcription factor E2F-1 and human telomerase reverse transcriptase (hTERT) promoters to control E1 offer some guarantee that not only is RB gene therapy effective, but it is also safe. Combination therapy using the oncolytic adenovirus Ad-TERT p-E1 and replication-defective adenovirus Ad-endostatin demonstrates desirable oncolysis in the in situ RB mouse model. Additionally, E1B19K is important in the RB tumor suppression effect of oncolytic adenoviruses.

\section{Introduction}

Retinoblastoma (RB) is the most common intraocular malignant tumor in infants, and RB is also common among children and adolescents (1). Though the RB patient survival rate is excellent with the application of current therapies, significant ocular damage often occurs that may have life-long impact on patient vision $(2,3)$. Over the past decades, treatment options for children with intraocular RB have dramatically changed. Attempts to avoid eye enucleation and beam radiotherapy have lead to increased use of globe-preserving techniques, including systemic chemotherapy, photocoagulation, brachytherapy, cryotherapy, and thermotherapy (4). Gene therapy provides valuable new options for the development of new RB treatments.

Human RB occurs primarily in either the familial or sporadic form. These two forms are both rooted in bi-allelic mutation of the RB gene (5), wherein local importation of the exogenous RB gene leads to irreversible growth arrest of tumor cell selectively (6). Previously, Jia et al (7) utilized small interference RNA (siRNA) for vascular endothelial growth factor (VEGF) to inhibit the formation of new blood vessels in RB models. Additionally, a phase I study has shown that delivery of the suicide gene (thymidine kinase) into RB tumors had effective oncolytic effects in vivo (8).

In contemporary $\mathrm{RB}$ research, oncolytic adenovirus treatment, alternatively referred to as conditionally replicating 
adenovirus (CRAd) treatment, has emerged as an innovative and promising platform for the treatment of many forms of cancer due to the ability of these vectors to replicate selectively in tumor cells, ultimately resulting in cell death by lysis of tumor cells $(9,10)$. Because viral replication is specific to tumor cells, the natural increase in local concentration of viral particles leads to the propagation of virus particles throughout the tumor. This may allow relatively low, non-toxic doses to be highly effective in elimination of entire tumors. A facilitation strategy that increases the selectivity of the oncolytic adenovirus is the use of tumor-specific promoters to control the expression of adenoviral genes essential for replication, such as E1A $(11,12)$. These promoters give priority to viral gene expression in tumor cells, limiting or entirely preventing damage to healthy tissues.

During tumorigenesis, the loss of the tumor suppressing retinoblastoma protein $(\mathrm{Rb})$ that binds to $\mathrm{E} 2 \mathrm{~F}$ leads to an apparent increase in free $\mathrm{E} 2 \mathrm{~F}$, or increasing E2f that exists independently of the Rb-E2F complex. The resultant abundance of free E2F, in turn, induces high-level expression of the E2F-responsive genes associated with retinoblastoma. Previously, E2F-1 overexpression has been observed in many other tumor cell lines, including specific lung and liver cancer lines. This occurrence is likely due to frequent disruptions in the $\mathrm{pRB} / \mathrm{p} 16 \mathrm{INK} 4 \alpha /$ cyclin $\mathrm{D}$ pathway. The $\mathrm{P} 16$ protein specifically binds to CDK4, which inhibits the kinase activity of CDK4. Conversely, Rb protein is phosphorylated by CDK4. In the absence of the P16 gene, the Rb protein is instead phosphorylated, and E2F-1 is released to prompt the transcription of its downstream target genes. Because these genes are essential to the transition to the S-phase, this disrupts the life cycle of normal cells (13-15). Thus, the human E2F-1 gene promoter is responsible for controlling the expression of key viral genes essential for replication, making it an excellent candidate for achieving specific tumor selectivity. E1 regulation in oncolytic adenoviruses by the E2F-1 promoter demonstrated high selectivity, indicating the precise level of control attainable by using the E2F-1 promoter (16). These findings led to the design of the study to explore an oncolytic adenovirus with E1A regulation controlled by the E $2 \mathrm{~F}-1$ promoter. This innovative design allowed for the investigation of the virus effectiveness in generating RB tumor cell death both in vitro and in vivo in mouse models in the current study for the first time.

Both the E1A and E1B genes are essential for adenovirus replication. E1A acts as a cue to initiate virus replication by activating the early adenovirus promoters, and it is also required to drive the host cell into the $S$-phase of the cell cycle for viral DNA replication (17). An adverse effect of E1A is that it stabilizes p53, which leads to apoptosis and is unfavorable for viral replication (18). To prevent this, E1B55K and the Ad E4 or f6 proteins form a complex with $\mathrm{p} 53$, causing its degradation through ubiquitin mediated proteolysis $(19,20)$. E1B19K additionally prevents E1A-induced apoptosis by interfering with the actions of the pro-apoptotic proteins Bak and $\operatorname{Bax}(21,22)$. It has been reported that the oncolytic adenovirus d11520 with E1B19K, as well as other E1B55K-deleted viruses, replicated efficiently in a variety of tumor cell lines independent of their p53 status (23-25). E1B19K deletion, however, has been indicated to generate more rapid viral release from apoptotic cells, resulting in enhancement to viral delivery across tumor tissues. Based on the high activity of telomerase reverse transcriptase (hTERT) in the human tumor cells, Doloff et al (26) constructed an oncolytic adenovirus, Ad-hTERT-E1A, with deletions of the viral E1B and E3 regions and an hTERT promoter-driven E1A cassette. This design possessed strong therapeutic potential as well as an improved safety profile compared to the previous d11520. Ji et al (27) constructed an oncolytic adenovirus with an E1A controlled using the hTERT promoter armed with the suicide gene thymidine kinase. This design demonstrated a good killing effect in RB tumor models in situ in the eyes of nude mice. The success of these designs provided the foundation for the current design, containing an oncolytic adenovirus inclusive of E1A and E1B19K controlled by hTERT promoter. The current design was evaluated for the anti-cancer effects of the viruses and the overall role of E1B in RB treatment.

Over the course of the current study, the targeted oncolytic adenovirus Ad-E2F1 p-E1A was constructed for RB treatment. In this design, E1A was controlled by the E2F1 promoter due to the notable fact that E2F-1 activity usually increases in RB cells. Additionally, the targeted oncolytic adenovirus Ad-TERT p-E1 was constructed, in which E1 was controlled by the TERT promoter. The replication capacity of these recombinant viruses and the effect to induce tumor cell death was studied in cancer cell lines HXO-RB44, Y79, Hep3B and NCIH460. Furthermore, simultaneous evaluation of the replication-defective adenovirus Ad-endostatin, carrying the human endostatin gene, revealed the anti-cancer efficacy of Ad-endostatin both in isolated treatments and when combined with Ad-E2F1 p-E1A or Ad-E1 TERT p-E1 treatments in situ for the treatment of RB tumor model nude mice. The results suggest that the gene-viral therapeutic system developed herein demonstrates the synergistic effects of viral oncolytic therapy and anti-angiogenesis therapy, generating a novel therapeutic strategy for human RB.

\section{Materials and methods}

Cell lines and cell culture. Cancer cell lines with Rb pathway defects used in the current study included the human lung cancer cell line NCIH460 (ATCC, Manassas, VA, USA) that is P16 negative, human hepatocyte cancer cell line Hep3B (ATCC), two human RB cell lines HXO-RB44 (Cancer Research Institute, Xiangya Medical College, Central South University of China) (28), and Y79 (Institute of Biochemistry and Cell Biology, Shanghai Institute of Biological Science, Chinese Academy of Science, Shanghai, China) that are Rb negative. Additionally selected were the human breast adenocarcinoma cell line MCF-7 that is Rb positive and the HLF human lung fibroblast cell line, both purchased from the Institute of Biochemistry and Cell Biology, Chinese Academy of Sciences. The human embryonic kidney cell line AD-293 used for packaging adenoviral vectors was purchased from Stratagene ( $\mathrm{La}$ Jolla, CA, USA). NCIH460, Hep3B, and AD-293 cells were cultured in Dulbecco's modified Eagle's medium (Gibco-BRL, Carlsbad, CA, USA) with 10\% fetal bovine serum. HXO-RB44 cells, HLF, and Y79 cells were maintained in ready mix RPMI1640 (Gibco-BRL) medium supplemented with 10\% fetal bovine serum. MCF-7 cells were cultured in RPMI-1640 with $10 \%$ fetal bovine serum and insulin $(0.01 \mathrm{mg} / \mathrm{ml})$. 
Table I. Primers used in oncolytic adenovirus construction.

Primers sequence

\begin{tabular}{|c|c|}
\hline E2F-1 promoter & $\begin{array}{ll}\text { F: } & \text { 5'-CCG GAA TTC CGG GGT ACC ATC CGG ACA AAG CCT GCG-3' } \\
\text { R: } & \text { 5'-CGC GGA TCC GCG CGA GGG CTC GAT CCC GCT CCG C-3' }\end{array}$ \\
\hline E1A gene (pE2F1 p-EGFP-1) & $\begin{array}{ll}\text { F: } & \text { 5'-CGG GAT CCA TGA GAC ATA TTA TCT GCC ACG-3' } \\
\text { R: } & \text { 5'-ATA AGA ATG CGG CCG CTT ATG GCC TGG GGC GTT TA-3' }\end{array}$ \\
\hline E1B gene & $\begin{array}{ll}\text { F: } & \text { 5'-TCC CCC GGG ATG GAG GCT TGG GAG TGT TT-3' } \\
\text { R: } & \text { 5'-GCT CTA GAT CAA TCT GTA TCT TCA TCG CT-3' }\end{array}$ \\
\hline E1A gene (pTERT p-IRES-E1B) & $\begin{array}{l}\text { F: 5'-CGG GAT CCG GGC CCA TGA GAC ATA TTA TCT GCC ACG-3' } \\
\text { R: 5'-CGG GAT CCT TAT GGC CTG GGG CGT TTA-3' }\end{array}$ \\
\hline
\end{tabular}

F, Forward; R, reverse.

Generation of HXO-RB44-GFP-Luc cells. HXO-RB44 cells were transfected with a plasmid carrying an enhanced green fluorescent protein (EGFP)-luciferase fusion gene expression cassette, kindly provided by Dr C.Y. Li (University of Colorado Health Sciences Center, USA) using Lipofectamine 2000 (Invitrogen, Carlsbad, USA). Positive transfectants were selected with $0.5 \mathrm{mg} / \mathrm{ml}$ Geneticin (Invitrogen). Single clones of positive transfectants were obtained by limited dilution. The stably transfected HXO-RB44-EGFP-Luc cells were maintained in RPMI-1640 medium containing $10 \%$ fetal bovine serum (FBS) and $0.1 \mathrm{mg} / \mathrm{ml}$ Geneticin. Clones expressing high levels of EGFP as well as luciferase were selected for further experiments.

Construction of recombinant oncolytic adenoviruses. A 269-bp fragment of human E2F-1 promoter (GenBank no. S74230) was amplified from human genomic DNA, and a 1011-bp fragment of the human adenoviral E1A gene (GenBank no. AC_000008) was amplified by polymerase chain reaction (PCR) from AD-293 cellular genomic DNA. The E1A gene and E2F-1 promoter were subcloned into pDC311 (Microbix Biosystems, Toronto, Canada), resulting in an adenoviral shuttle vector pDC311-E2F1 p-E1A that was subsequently co-transfected with the adenoviral backbone plasmid pBHGLox $\triangle \mathrm{E} 1 \mathrm{E} 3$ (Microbix Biosystems) into AD-293 cells to obtain oncolytic adenovirus Ad-E2F1 p-E1A.

A 1812-bp fragment of the human adenoviral E1B gene was amplified by PCR from the pAdE1-3 plasmid (kindly provided by Dr C.Y. Li), digested by $S m a \mathrm{I} / \mathrm{XbaI}$, and cloned into the pIRES-neo (Clontech) to obtain pIRES-neo-E1B, in which E1B55 kD was not expressed due to the deletion mutation of $\mathrm{G}$ 663. A 475-bp fragment containing the promoter of the human telomerase reverse transcriptase gene (GenBank no. NG_009265) was excised by EcoRI/BamHI from pTERT p-EGFP (kindly provided by Dr C.Y. Li) and inserted into pIRES-neo-E1B, resulting in pTERT p-IRES-E1B. The E1A gene was digested with $\mathrm{BamHI}$ and inserted into pTERT p-IRES-E1B to obtain pTERT p-E1A-IRES-E1B. Finally, an XhoI/NotI restriction fragment of pTERT p-E1A-IRES-E1B containing the hTERT promoter, E1A gene, E1B gene, and downstream poly A sequences was inserted into pENTR1A (Invitrogen). The resulting plasmid pENTR1A-TERT
p-E1A-IRES-E1B and adenoviral backbone plasmid pAd/ PL-DEST (Invitrogen) were LR recombination reacted. The homologously recombinant pAd/PL-DEST-TERTp-E1AIRES-E1B construct was then digested with PacI to expose the left and right viral ITRs and transfected into AD-293 cells to generate Ad-TERT p-E1. All primers used in adenovirus construction are listed in Table I, and the recombinant adenoviruses are illustrated in Fig. 1. The wild-type adenovirus d1309 containing a partial deletion of the E3 gene not affecting replication was prepared using an identical procedure to that described previously. This design was utilized as an oncolytic control. Ad-endostatin was a replication-deficient adenovirus containing endostatin (provided by Dr C.Y. Li). The replication-deficient adenoviruses Ad-null (with the deletion of both E1 and E3) and Ad-EGFP were preserved. Functional particle titers for all adenoviruses were determined by plaque assays in AD-293 cells and shown as plaque forming units/milliliter (pfu/ml).

Fluorescence microscopy observation and FACS analysis. Cells were seeded in 24-well plates one day before adenoviral infection. The following day, cells of $60-70 \%$ confluence were infected with Ad-EGFP either with or without oncolytic adenovirus at the indicated multiplicity of infection (MOI). After $72 \mathrm{~h}$, visualization of EGFP expression was carried out on a Zeiss Axio Uret S100 (Carl Zeiss Microscopy, LLC, USA) equipped with a Zeiss AxioCam color camera. In fluorescence activated cell sorting (FACS) analysis, cell fluorescence was measured using the FACSort with an excitation of $448 \mathrm{~nm}$ wavelength. Cell populations of interest were gated and analyzed using CellQuest ${ }^{\mathrm{TM}}$ software (Becton-Dickinson, USA).

Viral replication assays. HLF cells and log-phase tumor cells plated at $60-70 \%$ confluence were infected with Ad-E2F1 p-E1A or Ad-TERT p-E1 at an MOI of $10 \mathrm{pfu} / \mathrm{cell}$. Virus inocula were removed after a $4 \mathrm{~h}$ incubation period. The cells were then washed 3 times with sodium perborate (PBS) and incubated at $37^{\circ} \mathrm{C}$ for $48 \mathrm{~h}$. Cells were scraped into a $1 \mathrm{ml}$ medium, subjected to 3 freeze-thaw cycles, and centrifuged. The supernatant was subsequently collected. Serial dilutions of the supernatant were assayed for live virus particles by stan- 
dard plaque forming assays using AD-293 cells. The wild-type adenovirus d1309 was used as a control.

Western blot analysis. Tumor cells at $60-70 \%$ confluence were infected with different viruses at various MOIs. After $48 \mathrm{~h}$, cell extracts were prepared by lysis buffer containing $2 \%$ sodium dodecyl sulfate (SDS) and $0.125 \mathrm{M}$ Tris- $\mathrm{HCl}$ (pH 6.8). The total protein of the cell extracts was measured using the Bio-Rad Protein Assay kit (Bio-Rad, Hercules, USA). Proteins $(40 \mu \mathrm{g})$ were separated on $10 \%$ SDS-polyacrylamide gel and blotted onto a polyvinylidene difluoride membrane (Roche, Mannheim, Germany). The membrane was incubated with antibodies to E1A, and GAPDH (Santa Cruz Biotechnology Inc., USA) for $1 \mathrm{~h}$ at room temperature. After washing, the membrane was probed with the appropriate secondary peroxidase-conjugated antibodies and subsequently visualized using a chemiluminescence method (ECL, Roche). Images of the bands were captured using an image acquisition software system (ChemiDoc ${ }^{\mathrm{TM}} \mathrm{XRS}^{+}$; Bio-Rad).

In vitro cell viability assay. HXO-RB44-GFP-Luc cells and Y79 cells were plated at 50-60\% confluence in 96-well dishes and $24 \mathrm{~h}$ later infected with different adenoviruses at various MOIs. After 6 days, the cell viability was measured using the cell counting kit-8 (CCK-8) (Dojindo, Tokyo, Japan). Background refers to the absorbance of medium alone. The percentage of cell survival was calculated using the formula: $\%$ cell survival $=\left(\mathrm{A}_{490 \mathrm{~nm}}\right.$ of infected cells $-\mathrm{A}_{490 \mathrm{~nm}}$ of background $) /$ $\left(\mathrm{A}_{490 \mathrm{~nm}}\right.$ uninfected cells $-\mathrm{A}_{490 \mathrm{~nm}}$ of background $) \mathrm{x} 100 \%$.

In vivo antitumor effect. HXO-RB44-GFP-Luc cells were infected with various adenoviruses as follows: Ad-null 50 MOI, Ad-E2F1 p-E1A 12.5 MOI, Ad-TERT p-E1 12.5 MOI, Ad-endostatin 50 MOI, Ad-E2F1 p-E1A 12.5 MOI+Ad-endostatin 50 MOI, and Ad-TERT p-E1 12.5 MOI+Ad-endostatin 50 MOI. Virus inocula were removed after a $16 \mathrm{~h}$ incubation period. The cells were then prepared at $1 \times 10^{5}$ cells $/ \mu 1$ in PBS. RB tumors were established by injecting HXO-RB44-GFP-Luc cells into the vitreum of BALB/c (nu/nu) mice (Shanghai Laboratory Animal Center, Shanghai, China). A total of 10 mice were allocated to each group. Mice were anesthetized with $50 \mathrm{mg} / \mathrm{kg}$ pentobarbital intraperitoneally and a topical application of $0.4 \%$ oxybuprocaine. The pupil was dilated using an eye drop solution containing 0.5/0.5\% tropicamide/phenylephrine hydrochloride (Santen Pharmaceutical). A plastic ring filled with $2.5 \%$ cellulose was placed on the cornea to assist in visualizing the fundus. The injection was performed under a binocular surgical microscope.

A total of $2 \times 10^{5}$ cells prepared in $2 \mu \mathrm{l}$ PBS were injected slowly into the midvitreous of the left eye with a $32 \mathrm{G}$ needle attached to a $10 \mu \mathrm{l}$ microsyringe. The bioluminescent image of the HXO-RB44-GFP-Luc tumor in the vitreous was acquired by in vivo bio-layer interferometry (BLI) technology using a NightOwl LB 981 Molecular Imaging System (Berthold Technologies, Bad Wildbad, Germany) on Day 15 after injection. Each subject was injected i.p. with $100 \mathrm{mg} / \mathrm{kg}$ D-luciferin (Molecular Imaging Products, Ann Arbor, MI, USA) in $100 \mu 1$ PBS, anesthetized with pentobarbital, and images were acquired after $5 \mathrm{~min}$. The exposure time of the bioluminescent images was set to $10 \mathrm{~min}$. Images were processed and pseu- docolored using WinLight software (Berthold Technologies). Alternatively, tumor growth was determined by fluorescence signal on Day 20 after injection by direct stereomicroscopy (Stemi SV11; ZEISS, Jena, German).

The onset of EGFP gene expression and its distribution in the vitreous region was investigated. Interesting regional optical density $(\mathrm{IOD})=$ eye area $\left(\mu \mathrm{m}^{2}\right) \mathrm{x}$ mean fluorescence intensity (grey)/2(16) bit. All animal subjects were sacrificed 42 days post-injection, and eyes were enucleated for pathological examination. All mice were maintained and handled in accordance with the guidelines approved by national and local institutions.

Histopathology. Mouse eyes were fixed in $10 \%$ formalin and embedded in paraffin. The sections were routinely stained with hematoxylin and eosin $(\mathrm{H} \& \mathrm{E})$ and blindly evaluated by two individual pathologists.

Statistical analysis. All in vitro experiments were completed three times under separate conditions, and the in vitro and in vivo experimental data are presented as the mean plus or minus standard deviation (mean $\pm \mathrm{SD}$ ). Comparisons were made using ANOVA with appropriate post-hoc tests (Fisher's PLSD). Survival was analyzed by the Kaplan-Meier method, and results were compared for statistical significance using the generalized Wilcoxon test. All statistical analyses were performed using SPSS10.0 software. P-values <0.05 were considered statistically significant.

\section{Results}

Construction of recombinant oncolytic viruses. Two types of conditionally replicating adenoviruses (CRAds), Ad-E2F1 p-E1A and Ad-TERT p-E1, were developed. In these designs, the E1A of Ad-E2F1 p-E1A was under the control of E2F1 promoter and the E1A and E1B19K of Ad-TERT p-E1 were under the control of human telomerase reverse transcriptase (hTERT) promoter (Fig. 1). Absence of the wild-type virus was confirmed by PCR for the E3 gene (data not shown).

Selective replication of the two recombinant oncolytic adenoviruses. In order to demonstrate the selective replication of the Ad-E2F1 p-E1A vector, cancer cell lines HXO-RB44, Y79, Hep3B, NCIH460, MCF-7, and HLF cells were infected using Ad-E2F1 p-E1A. The resultant production of viral particles was quantified with the plaque forming assay method (Fig. 2A). The result showed that Ad-E2F1 p-E1A replicated well in HXO-RB44, Y79, Hep3B and NCIH460 tumor cells in which the Rb pathway was dysregulated, but not in MCF-7 and HLF cells that are $\mathrm{Rb}$ positive. Ad-TERT $\mathrm{p}$-E1 replicated remarkably well in tumor cells HXO-RB44, Y79, Hep3B, NCIH460 and MCF-7, but not in HLF cells. Moreover, Ad-TERT p-E1 replicated more efficiently in Hep3B, NCIH460, and MCF-7 cells than Ad-E2F1 p-E1A $(\mathrm{P}<0.05)$ (Fig. 2A). Selective replication of the Ad-E2F1 p-E1A was further confirmed by western blot analysis, as the E1A protein was only expressed in HXO-RB44, Y79, Hep3B and NCIH460 tumor cells (Fig. 2B).

CRAds for activating expression of the GFP transgene in cancer cells. In order to assess the feasibility and effective- 


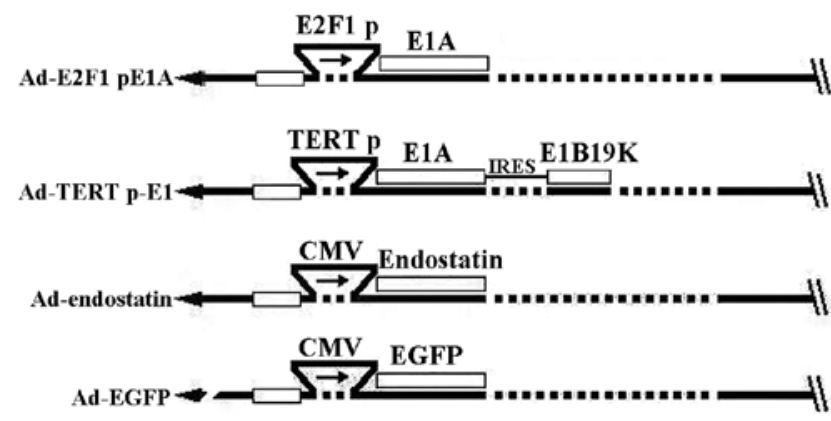

Figure 1. Schematic illustration of the adenoviruses used in this study. These 4 types of adenoviruses were constructed based on E1,E3-deleted adenovirus. For Ad-E2F1 p-E1A, the E1A expression cassette controlled by the E2F1 promoter was inserted into the E1 region. For Ad-TERTp-E1, the expression cassette of E1A and E1B19K connected by IRES was transcribed under the control of the hTERT promoter and inserted into the E1 region, but E1B55K deleted. Human endostatin or EGFP expression cassette controlled by the cytomegalovirus promoter was inserted into the E1 region to generate Ad-endostatin or Ad-EGFP respectively. IRES, the internal ribosome entry site sequence.

ness of the strategy involving non-replicating adenovirus replication driven by a replicative adenovirus, the fluorescence intensity was observed when an oncolytic adenovirus was administered along with Ad-EGFP. Both Ad-E2F1 p-E1A and Ad-TERT p-E1 showed significantly improved EGFP-positive rates and mean fluorescence intensity in HXO-RB44 cells and Y79 cells. Furthermore, the average fluorescence intensity of EGFP in Hep3B cells increased, but Ad-E2F1 p-E1A reduced EGFP expression in the tumor cell line MCF-7 with normal $\mathrm{Rb}$ status $(\mathrm{P}<0.001)($ Fig. 3A). The percentage of EGFP-positive HXO-RB44 cells increased from $25.7 \pm 2.30 \%$ (25 MOI of Ad-EGFP) to $44.40 \pm 6.20 \%$ (25 MOI of Ad-E2F1 p-E1A and $25 \mathrm{MOI}$ of Ad-EGFP) $(\mathrm{P}<0.05)$, a value close to the level of 500 MOI Ad-EGFP $(45.0 \pm 4.90 \%)$ (Fig. 3B). The EGFP-positive rate was raised to $84.75 \pm 0.65 \%$ and $91.10 \pm 0.10 \%$, respectively, when Ad-EGFP was co-administered with $100 \mathrm{MOI}$ and $250 \mathrm{MOI}$ of Ad-E2F1 p-E1A. This was significantly higher than that of the same amount of Ad-TERT p-E1 $(\mathrm{P}<0.001)$. Moreover, Ad-E2F1 p-E1A (100, 250 and 500 MOI) elevated the mean fluorescence intensity of EGFP to a significantly higher level than the same MOI of Ad-TERT p-E1 ( $\mathrm{P}<0.01)$. Similarly, a combination of Ad-E2F1 p-E1A with Ad-EGFP resulted in a higher EGFP-positive rate than that of Ad-TERT p-E1 plus Ad-EGFP in Y79 cells $(\mathrm{P}<0.001)$ (Fig. 3C). These results suggested that the oncolytic adenovirus was efficient in improving the expression of the exogenous gene carried by non-replicating adenovirus.

Cell-killing effect in vitro of Ad-E2F1 p-E1A and Ad-TERT $p-E 1$ on $R B$ cells. The results of CKK-8 assay showed that when HXO-RB44 cells were infected with Ad-TERT p-E1 at 50,100 and $200 \mathrm{MOI}$, the cell survival rate was $62.08 \pm 9.82 \%$ $(\mathrm{P}<0.05), 20.59 \pm 4.75 \%(\mathrm{P}<0.001)$ and $11.32 \pm 2.54 \%(\mathrm{P}<0.001)$, respectively. The oncolytic effect of Ad-TERT p-E1 at 100 and $200 \mathrm{MOI}$ were significantly better than those observed in d1309 and Ad-E2F1 p-E1 ( $\mathrm{P}<0.001$, respectively). In addition, the oncolytic effect of dl309, Ad-E2F1 p-E1A and Ad-TERT p-E1 on Y79 cells were not obvious. Ad-TERT p-E1 and Ad-E2F1 p-E1A combined did exhibit a significant treatment difference $(\mathrm{P}<0.05)$ (Fig. 3D).

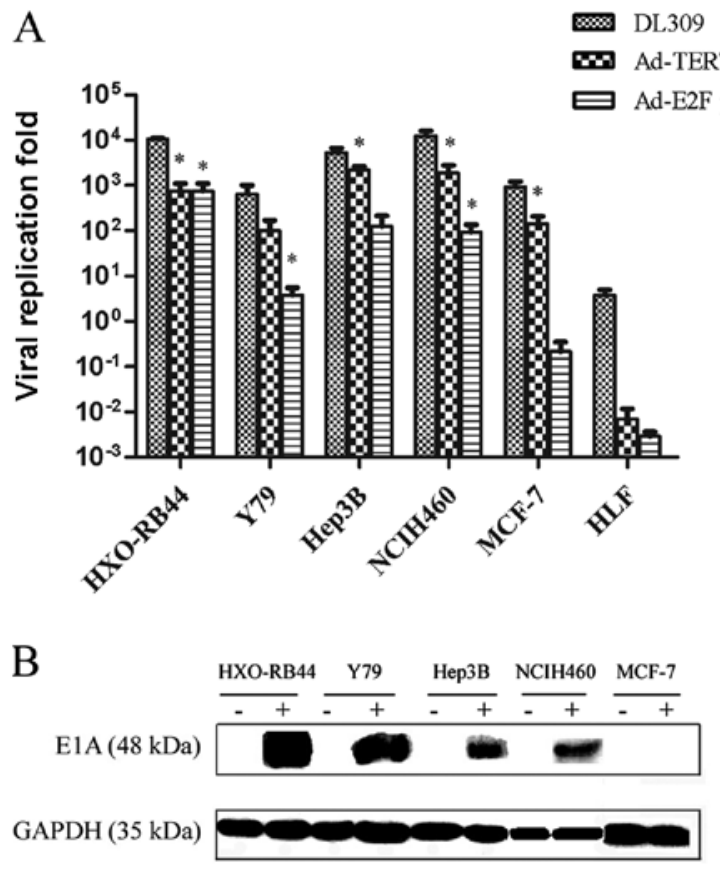

Figure 2. Selective replication of oncolytic adenovirus in vitro. (A) The cancer cell lines HXO-RB44, Y79, Hep3B, NCIH460, MCF-7 and the normal HLF lung fibroblast cells were infected with recombinant oncolytic adenoviruses Ad-TERT p-E1 or Ad-E2F p-E1A at a MOI of 10 pfu/cell, with wild-type adenovirus d1309 as a control. The cell lysates were prepared at $48 \mathrm{~h}$ and viral titers were measured by plaque forming assay method. The titer data at $48 \mathrm{~h}$ were normalized with those at the beginning of infection and shown as fold of replication. Values shown are mean \pm SD for triplicate samples. The replication multiples of oncolytic adenovirus in cancer cells were significantly higher than in HLF cells ( $\mathrm{P}<0.05)$. (B) Representative immunoblot for E1A protein (48 kDa) expression of cancer cell lines HXO-RB44, 79, Hep3B, NCIH460, MCF-7 infected with Ad-E2F p-E1A (+) at a MOI of $20 \mathrm{pfu} / \mathrm{cell}$ $48 \mathrm{~h}$ later. Uninfected cancer cells were used as negative control respectively (-). Bottom panel shows the GAPDH (35 kDa) control.

Antitumor efficacy of adenovirus for in situ RB in nude mice. The vitreous bodies of nude mice were inoculated with $2 \times 10^{5}$ HXO-RB44-GFP-Luc cells that were infected with the above adenoviruses, respectively. The anti-RB effect of oncolytic adenovirus on in vivo cells were measured. On Day 15 , in vivo imaging of the fundus was performed by intraperitoneal injection of a luciferase substrate. Compared with Ad-E2F1 p-E1A, Ad-TERT p-E1 was shown to inhibit tumor growth more effectively, and Ad-TERT p-E1+Ad-endostatin almost eliminated the tumor completely (Fig. 4C). Fluorescence signals in the nude mouse retina were investigated by fluorescence stereoscope on Day 20 (Fig. 4A). Compared with Ad-null, Ad-E2F1 p-E1A showed no significant difference in RB tumor cell death $(\mathrm{P}>0.05)$, while stronger effects were observed in the Ad-TERT p-E1, Ad-endostatin, Ad-E2F1 p-E1A+Ad-endostatin, and Ad-TERT p-E1+Adendostatin groups (all $\mathrm{P}<0.05$ ) (Fig. 4B). Co-administration of Ad-endostatin and Ad-TERT p-E1 generated a stronger oncolytic effect than Ad-endostatin ( $\mathrm{P}=0.037)$, comparable to that of Ad-TERT p-E1 ( $\mathrm{P}=0.097)$ (data not shown). Notably, only one of the 10 mice in the Ad-endostatin+Ad-TERT p-E1 group subsequently developed $\mathrm{RB}$. These results suggest that the oncolytic adenovirus and Ad-endostatin combination synergistically cause cell death in cancerous RB cells in nude mice. 


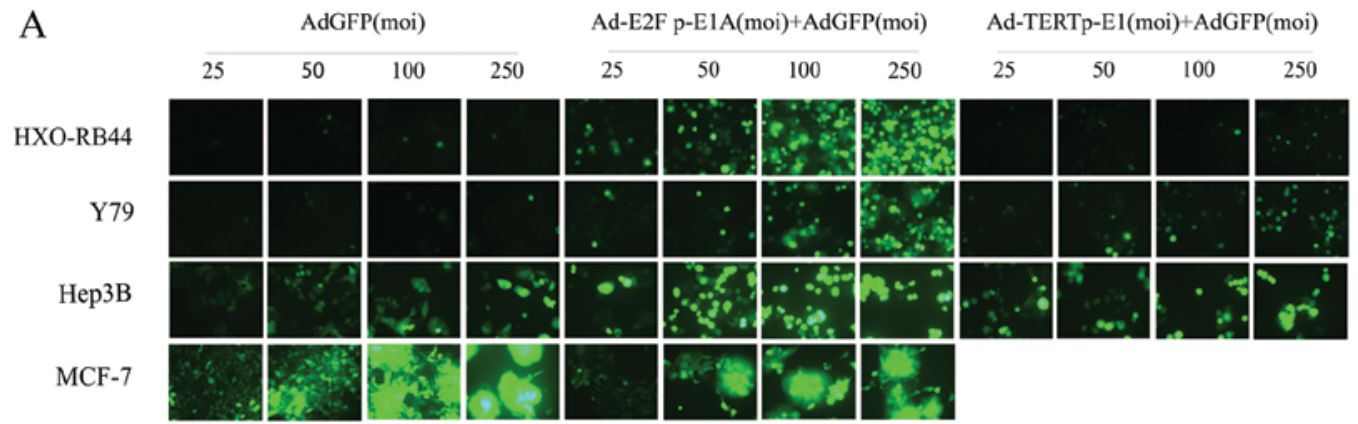

B HXO-RB44

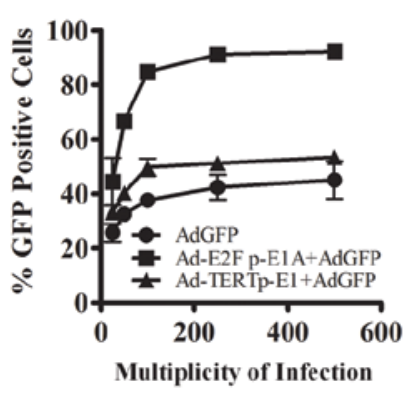

C $\quad$ Y79

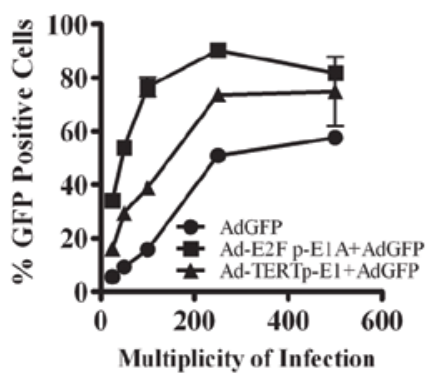

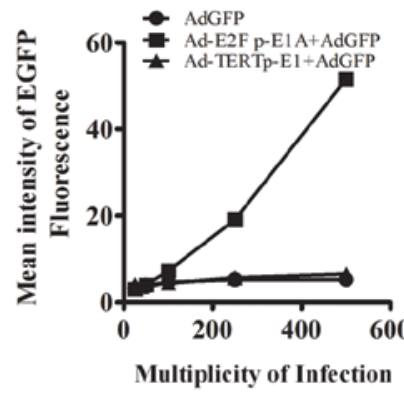

D HXO-RB44
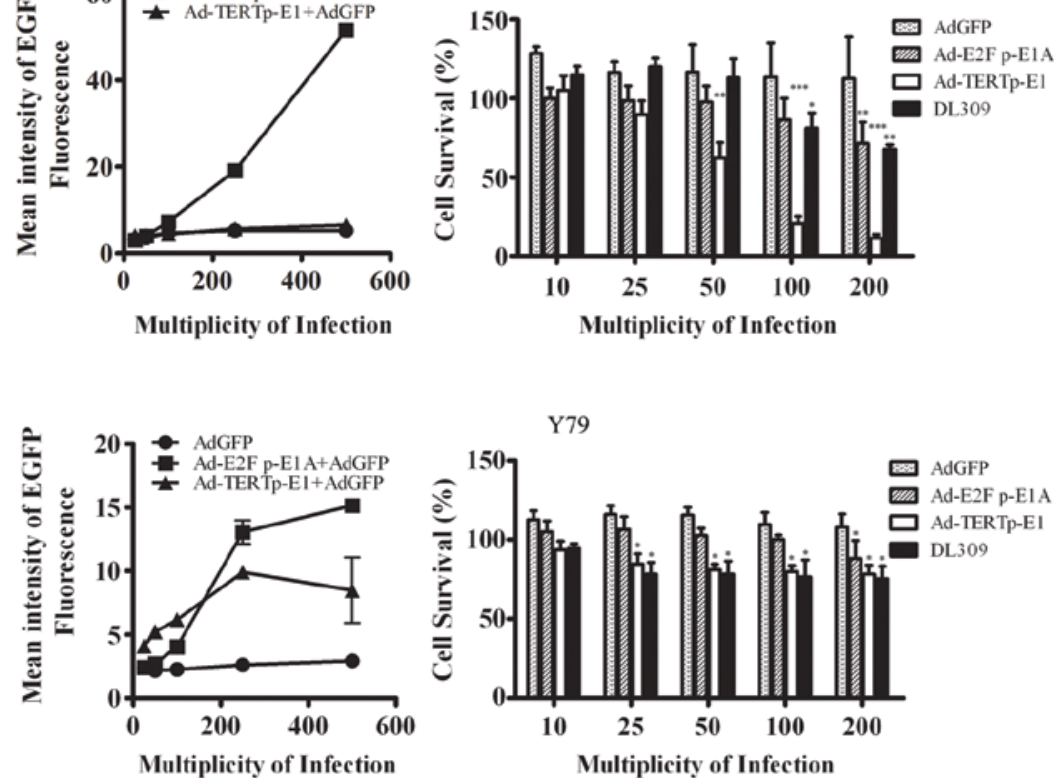

Y79

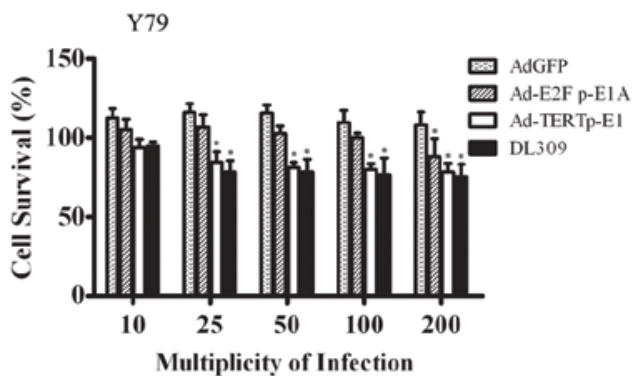

Figure 3. The ability of oncolytic adenovirus to activate expression of the GFP transgene in cancer cells. (A) HXO-RB-44,Y79, Hep3B and MCF-7 cells were infected with Ad-GFP at a MOI of 25, 50, 100, 250 pfu/cell, combined with a same amount of Ad-E2F p-E1A or Ad-TERT p-E1 or not. At 3 days post-infection, cells were observed and photographed by fluorescent microscope (Zeiss, Axio 100, original magnification, x400). (B) FACS analysis of average percentage and mean fluorescent intensity in HXO-RB44 cells at 3 days post-infection by viral vectors. (C) FACS analysis of average percentage and mean fluorescent intensity in Y79 cells at 3 days post-infection by viral vectors. The average percentage and mean fluorescent intensity were calculated from 3 individual wells for each viral vector infection and at least 3 repeated infection. (D) HXO-RB44 cells and Y79 cells were infected with Ad-GFP or Ad-E2F p-E1A or Ad-TERTp-E1 or the wild-type control virus d1309 at an MOI of 10, 25, 50, 100, 200 pfu/cell respectively. Cell viability was measured using CCK-8 assay 7 days post-infection. All experiments were performed in triplicates and repeated 3 times. Data are shown as the mean \pm SD. ${ }^{*}<0.05$, ${ }^{* *} \mathrm{P}<0.01$ and ${ }^{* * *} \mathrm{P}<0.001$.

Effect of oncolytic adenovirus on the survival time of nude mice with in situ RB. H\&E staining revealed that tumor cell number in the vitreous body was consistent with the results of green fluorescence previously identified by IOD signal quantitative analysis (Fig. 5A). The average survival times of the Ad-null group, Ad-E2F1 p-E1A group, Ad-TERT p-E1 group, Ad-endostatin group, Ad-E2F1 p-E1A+Ad-endostatin group, and Ad-TERT p-E1+Ad-endostatin group were 21 \pm 0.19 , $20 \pm 4.24,24 \pm 3.74,38 \pm 0,38 \pm 5.66$ and $42 \pm 0$ days, respectively. The survival rate in Ad-TERT p-E1+Ad-endostatin group was higher than that of Ad-TERT p-E1 group $(\mathrm{P}=0.022)$ and Ad-endostatin group $(\mathrm{P}=0.006)$, indicating that the Ad-TERT p-E1 and Ad-endostatin worked synergistically. These results revealed that oncolytic viruses with Ad-endostatin did significantly extend the survival time of mice with RB tumors, though oncolytic virus treatment alone failed to improve survival (Fig. 5B).

\section{Discussion}

A feasible solution to overcome the limitations in capacity of recombinant adenovirus treatments, which have previously limited the effectiveness of these treatments, is described. This solution utilizes oncolytic adenovirus-driven expression of therapeutic genes armed with non-replicating adenoviruses, a novel combination approach. In this method, E1A and E1B provided by oncolytic adenoviruses in tumor cells enable the replication of a non-replicating adenovirus, greatly increasing the quantity of copies of the therapeutic gene without adverse affects to living tissues. The advantage of this strategy is that one kind of oncolytic adenovirus can be combined with any other non-replicating viruses carrying therapeutic genes, or even multiple non-replicating viruses, despite the capacity limit of adenovirus packaging. This strategy is especially useful for individual gene therapy. Successful combination therapy using 
A
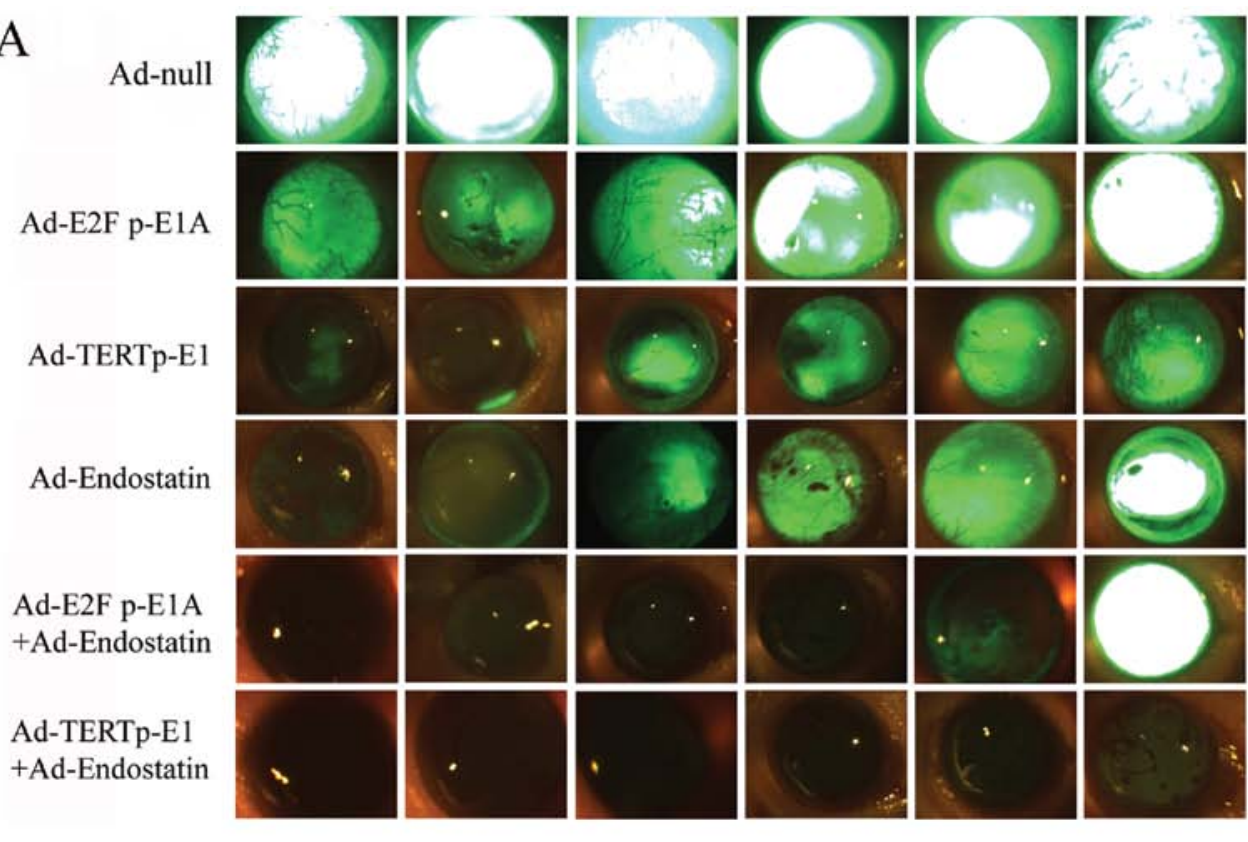

B
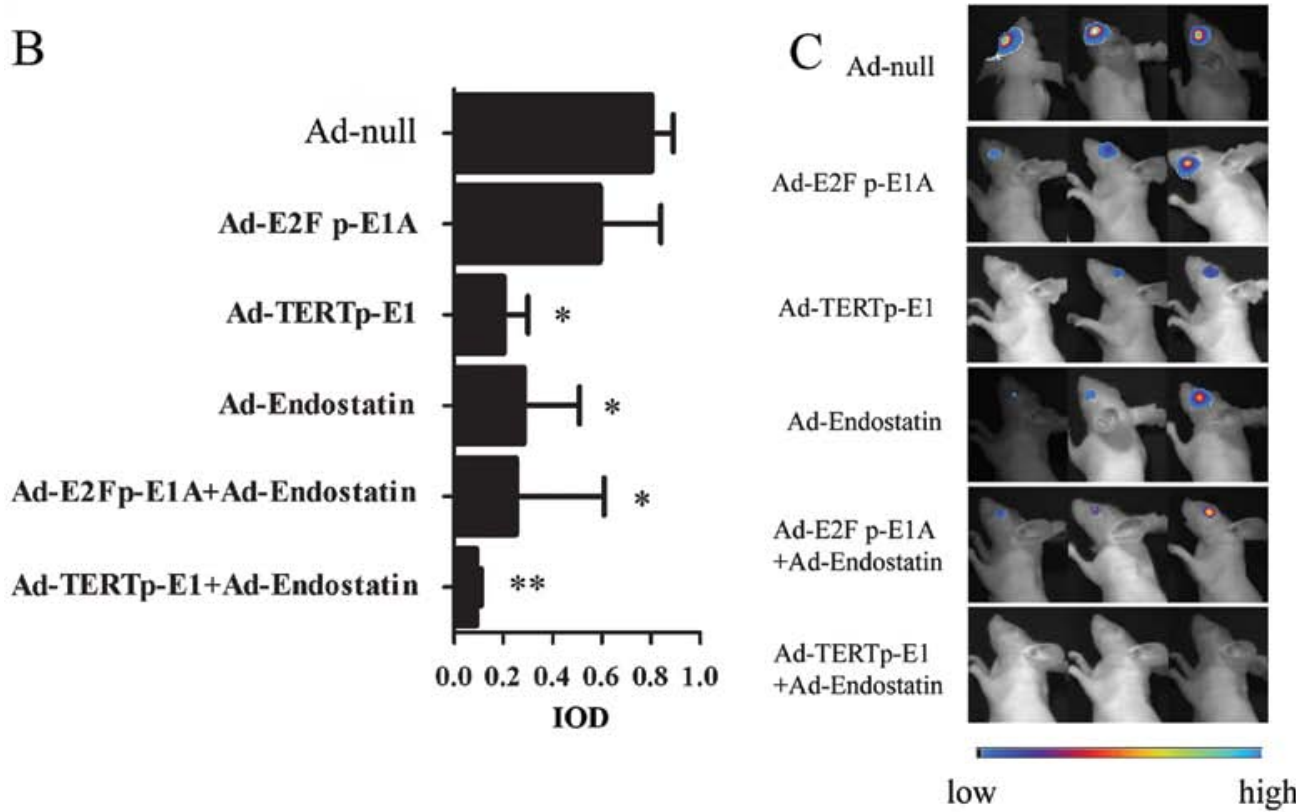

low high

Figure 4. Antitumor efficacy in HXO-RB44-GFP-Luc in a mouse tumor model. HXO-RB44-GFP-Luc cells (1x10 ${ }^{7}$ ) were infected with Ad-null (50 MOI), Ad-E2F p-E1A (12.5 MOI), Ad-TERTp-E1 (12.5 MOI), Ad-endostatin (50 MOI), Ad-E2Fp-E1A (12.5 MOI) + Ad-endostatin (50 MOI), Ad-TERTp-E1 $(12.5 \mathrm{MOI})+$ Ad-endostatin $(50 \mathrm{MOI})$ respectively. After $16 \mathrm{~h}$ infection, $2 \times 10^{5} \mathrm{HXO}-\mathrm{RB} 44-\mathrm{GFP}-$ Luc cells in $2 \mu 1$ PBS were injected into the vitreous body of $\mathrm{BALB} / \mathrm{c}(\mathrm{nu} / \mathrm{nu})$ mice, with 10 mice in each of the groups. (A) Fluorescence images of HXO-RB44-GFP-Luc tumors in vivo 20 days later. (Zeiss, StemiSV11, original magnification, $\mathrm{x} 32$ ) (B) Comparison of fluorescence signaling between groups. The tumor growth is determined with IOD (interesting regional optical density) using Axiovision 3.1 software quantitative methods. The data are shown as the mean $\pm \mathrm{SD}$. ${ }^{*} \mathrm{P}<0.05,{ }^{* * *} \mathrm{P}<0.01$. (C) Representative BLI images of RB tumors in each group at day 15. (NightOwl LB 981 Molecular Imaging System; Berthold Technologies).

an oncolytic virus along with an anti-angiogenesis gene carried by the Ad-endostatin for the treatment of RB was reported for the first time in the current study. The treatment achieved potent synergistic antitumor effects and extended the survival time of nude mice exhibiting RB tumors, demonstrating promising future prospects for RB treatment in human patients.

The oncolytic virus Ad-E2F1 p-E1A selectively replicated in HXO-RB44 cells with Rb gene deletion as well as in Hep3B and NCIH460 cells with inactivation of the P16 pathway. Similar results were not observed, however, in MCF-7 or HLF cells that possess a much lower E2F1 activity. Similarly, Ad-TERT p-E1 replicated efficiently in each of the tumor cell lines described above, with the exception of normal human cell HLF. Despite the observed activity from each virus treatment, notable differences were observed between Ad-E2F1 p-E1A and Ad-TERT p-E1 in replication and oncolytic efficiency. The number of Ad-TERT p-E1 progeny virus in tumor cells of Hep3B, NCIH460, and MCF-7 were 17-, 20-, and 642-foldhigher than those observed in Ad-E2F1 p-E1A, respectively $($ all $\mathrm{P}<0.05)$. E1A protein extended the half-life of $\mathrm{p} 53$ protein, in turn promoting premature apoptosis of the tumor cell and limiting the viral replication ability of Ad-E2F1 p-E1A (18). The E1B19K expressed by Ad-TERT p-E1, a functional Bcl-2 homologue, directly binds Bax, Bak-inhibiting oligomeriza- 


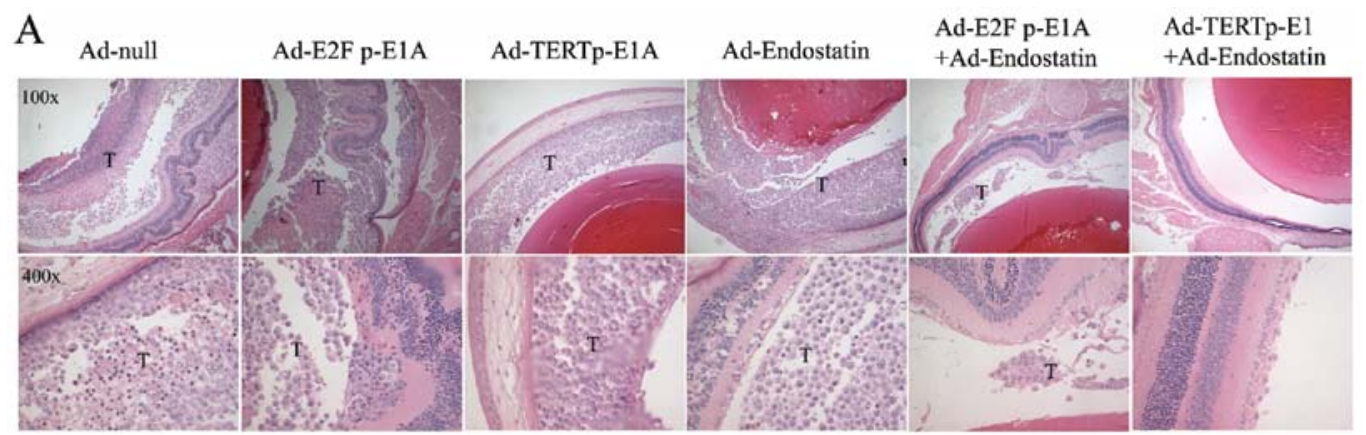

B

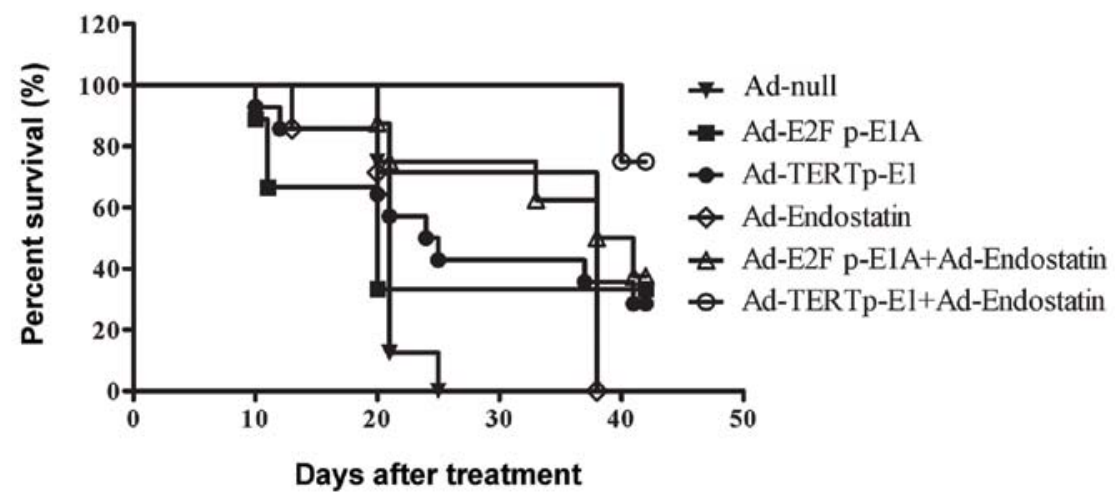

Figure 5. (A) Representative optical RB tumor section stained with H\&E in different groups treated with CRAd and endostatin gene therapy. T, tumor (original magnification, top x100, bottom x400). (B) Kaplan-Meier survival analysis of mice with established RB tumor in different groups after treatment with oncolytic adenovirus and endostatin gene.

tion, and mitochondrial pore-formation resulting in apoptosis blockage $(29,30)$. The biological function of E1B19K is to inhibit receptor-induced signaling at the time of cell death by preventing the Bax-Bak association, thus intrinsically inhibiting induced apoptosis through the p53-dependent and p53-independent mechanisms. The effect can be observed, for example, in the cellular response to viral E1A proteins (31-34). The anti-apoptotic E1B19K protein promotes viral replication, allowing it to propagate throughout the tumor. Matsushita et al (35) also found that adeno-associated virus production would be reduced by at least 100-fold when adenovirus-bearing mutated E1B19K was used as a helper virus. Polster et al (36) found that the number of progeny virus produced by the E1B19K mutated oncolytic virus was 10-fold lower than that produced by intact E1B19K (36). Our results have an important common similarity with the previous results.

The in vitro ability of Ad-TERT p-E1 to induce targeted RB cell death was significantly higher than that of Ad-E2F1 p-E1A $(\mathrm{P}<0.01)$. There are notable differences in the structure of Ad-TERT p-E1 and Ad-E2F1 p-E1A that may play a role in this activity difference. In each, different promoters were used to regulate $\mathrm{E} 1$ or $\mathrm{E} 1 \mathrm{~A}$ and the $\mathrm{E} 1$ region of the wild-type adenovirus consists of E1A, E1B19K and E1B55K, while the Ad-TERT p-E1 includes only E1A and E1B19K and Ad-E2F1 p-E1A includes only E1A. The ability of the Ad-hTERTE1A-CMV-HSVtk oncolytic virus to induce RB cell death was previously reported by the authors in the absence of the prodrug ganciclovir (GCV) on HXO-RB44 cells in vitro. Additionally, an in vivo RB model showed comparable results to that of Ad-E2F1 p-E1A (27), though results were significantly weaker than those of Ad-TERT p-E1. Tsukuda et al (37) and Jakubczak et al (16) also observed potent oncolytic effects of an oncolytic adenovirus containing the whole intact adenoviral $\mathrm{E} 1$ region controlled by the $\mathrm{E} 2 \mathrm{~F} 1$ promoter in tumor cell lines A549, HeLa, and SKOV-3 with elevated E2F1 activity. Based on these observations, the composition of the adenovirus E1 region is likely to play a key role in the adenovirus oncolytic effect, while tumor-specific promoters of adenoviral genes may merely correlate with the selectivity and safety of the virus. Ultimately, it is the E1B19K protein, not the promoters, that results in the difference in oncolytic efficiency observed in Ad-TERT p-E1 and Ad-E2F1 p-E1A.

When combined with replication-defective virus Ad-EGFP, both oncolytic virus Ad-TERT p-E1 and Ad-E2F1 $\mathrm{p}-\mathrm{E} 1 \mathrm{~A}$ produced elevation in average fluorescence intensity of EGFP and in the EGFP-positive rate. This effect was most significant in HXO-RB44 and Y79 cell lines, though the fluorescence intensity was also increased in Hep3B cells. Moreover, Ad-E2F1 p-E1A was superior to Ad-TERT p-E1 in promoting expression of the exogenous gene, consistent with previous reports (38). In HXO-RB44 cells, Ad-TERT p-E1 was likely to have induced greater RB tumor cell death when co-administered with Ad-EGFP. These results explain the observation that Ad-TERT $\mathrm{p}$-E1 produced inferior results to those observed in Ad-E2F1 p-E1A in promoting the expression of EGFP.

Compared with Ad-E2F1 p-E1A, Ad-TERT p-E1 demonstrated better tumor-targeting and ability to induce $\mathrm{Rb}$ tumor cell death. The combination of Ad-TERT p-E1 and Ad-endostatin led to an even more potent anti-cancer effect on RB mouse models in situ, resulting in a longer survival time than either the oncolytic virus or Ad-endostatin administered in isolation. The strategy of replicative adenovirus driven replica- 
tion of non-replicating adenoviruses has been achieved in the combination of the oncolytic virus and anti-angiogenesis gene. This demonstrates great advantages as well as promising prospects for future treatment of RB tumors in humans. In order to achieve the goal of more effective clinical RB treatments, the appropriate tumor-specific or tissue-specific promoter to control the replication of oncolytic adenovirus must be identified. Additionally, assessment and improvement in the safety of gene therapy must be made. The choice to retain E1B19K in the oncolytic adenovirus proved to be essential for ideal RB oncolysis, and it provides a basis for future development of combination gene treatments using adenoviruses for cancer treatment.

\section{Acknowledgements}

This study was supported by the National Science Foundation of China (nos. 30500553, 30672440). We would like to thank Professor Qian Huang for her contribution to this study and technical assistance. Additionally, we would like to gratefully acknowledge Yufei Wang and the entire staff at the Central Experimental Laboratory of Shanghai Jiaotong University Affiliated First People's Hospital.

\section{References}

1. Lohmann DR and Gallie BL: Retinoblastoma: revisiting the model prototype of inherited cancer. Am J Med Genet C Semin Med Genet 129C: 23-28, 2004.

2. Gatta G, Capocaccia R, Stiller C, Kaatsch P, Berrino F and Terenziani M; EUROCARE Working Group: Childhood cancer survival trends in Europe: a EUROCARE Working Group study. J Clin Oncol 23: 3742-3751, 2005.

3. Abramson DH, Beaverson K, Sangani P, et al: Screening for retinoblastoma: presenting signs as prognosticators of patient and ocular survival. Pediatrics 112: 1248-1255, 2003.

4. Balmer A, Zografos L and Munier F: Diagnosis and current management of retinoblastoma. Oncogene 25: 5341-5349, 2006.

5. Sun A, Bagella L, Tutton S, Romano G and Giordano A: From G0 to S phase: a view of the roles played by the retinoblastoma $(\mathrm{Rb})$ family members in the Rb-E2F pathway. J Cell Biochem 102: 1400-1404, 2007.

6. Sabado Alvarez C: Molecular biology of retinoblastoma. Clin Transl Oncol 10: 389-394, 2008.

7. Jia RB, Zhang P, Zhou YX, et al: VEGF-targeted RNA interference suppresses angiogenesis and tumor growth of retinoblastoma. Ophthalmic Res 39: 108-115, 2007.

8. Chevez-Barrios P, Chintagumpala M, Mieler W, et al: Response of retinoblastoma with vitreous tumor seeding to adenovirusmediated delivery of thymidine kinase followed by ganciclovir. J Clin Oncol 23: 7927-7935, 2005.

9. Kirn D, Martuza RL and Zwiebel J: Replication-selective virotherapy for cancer: Biological principles, risk management and future directions. Nat Med 7: 781-787, 2001.

10. Kruyt FA and Curiel DT: Toward a new generation of conditionally replicating adenoviruses: pairing tumor selectivity with maximal oncolysis. Hum Gene Ther 13: 485-495, 2002.

11. Ramachandra M, Rahman A, Zou A, et al: Re-engineering adenovirus regulatory pathways to enhance oncolytic specificity and efficacy. Nat Biotechnol 19: 1035-1041, 2001.

12. Fuerer $C$ and Iggo R: Adenoviruses with Tef binding sites in multiple early promoters show enhanced selectivity for tumour cells with constitutive activation of the wnt signalling pathway. Gene Ther 9: 270-281, 2002.

13. Sherr CJ: Cancer cell cycles. Science 274: 1672-1677, 1996.

14. Strauss M, Lukas J and Bartek J: Unrestricted cell cycling and cancer. Nat Med 1: 1245-1246, 1995.

15. Lu K, Shih C and Teicher BA: Expression of pRB, cyclin/cyclindependent kinases and E2F1/DP-1 in human tumor lines in cell culture and in xenograft tissues and response to cell cycle agents. Cancer Chemother Pharmacol 46: 293-304, 2000.
16. Jakubczak JL, Ryan P, Gorziglia M, et al: An oncolytic adenovirus selective for retinoblastoma tumor suppressor protein pathway-defective tumors: dependence on E1A, the E2F-1 promoter, and viral replication for selectivity and efficacy. Cancer Res 63: 1490-1499, 2003.

17. Ries SJ and Brandts $\mathrm{CH}$ : Oncolytic viruses for the treatment of cancer: current strategies and clinical trials. Drug Discov Today 9: 759-768, 2004

18. Lowe SW, Ruley HE, Jacks T and Housman DE: p53-dependent apoptosis modulates the cytotoxicity of anticancer agents. Cell 74: 957-967, 1993.

19. Querido E, Marcellus RC, Lai A, et al: Regulation of p53 levels by the E1B 55-kilodalton protein and E4orf6 in adenovirusinfected cells. J Virol 71: 3788-3798, 1997.

20. Steegenga WT, Riteco N, Jochemsen AG, Fallaux FJ and Bos JL: The large E1B protein together with the E4orf6 protein target p53 for active degradation in adenovirus infected cells. Oncogene 16: 349-357, 1998.

21. Cuconati A, Degenhardt K, Sundararajan R, Anschel A and White E: Bak and Bax function to limit adenovirus replication through apoptosis induction. J Virol 76: 4547-4558, 2002.

22. Farrow SN, White JH, Martinou I, et al: Cloning of a bcl-2 homologue by interaction with adenovirus E1B 19K. Nature 374: 731-733, 1995.

23. Hobom U and Dobbelstein M: E1B-55-kilodalton protein is not required to block p53-induced transcription during adenovirus infection. J Virol 78: 7685-7697, 2004.

24. Rao XM, Tseng MT, Zheng X, et al: E1A-induced apoptosis does not prevent replication of adenoviruses with deletion of E1b in majority of infected cancer cells. Cancer Gene Ther 11: 585-593, 2004.

25. Rao XM, Zheng X, Waigel S, Zacharias W, McMasters KM and Zhou HS: Gene expression profiles of normal human lung cells affected by adenoviral E1B. Virology 350: 418-428, 2006.

26. Doloff JC, Waxman DJ and Jounaidi Y: Human telomerase reverse transcriptase promoter-driven oncolytic adenovirus with E1B-19 kDa and E1B-55 kDa gene deletions. Hum Gene Ther 19: 1383-1400, 2008.

27. Ji X, Zhang J, Cheng L, et al: Oncolytic adenovirus delivering herpes simplex virus thymidine kinase suicide gene reduces the growth of human retinoblastoma in an in vivo mouse model. Exp Eye Res 89: 193-199, 2009.

28. Xu H, Wang C, Zhu H, Liu S, Xu X and Jiang Y: Characteristics of an established retinoblastoma cell line $\mathrm{HXO}-\mathrm{Rb} 44$. Yan $\mathrm{Ke}$ Xue Bao 11: 16-21, 1995.

29. White E: Regulation of the cell cycle and apoptosis by the oncogenes of adenovirus. Oncogene 20: 7836-7846, 2001.

30. Subramanian T, Vijayalingam S and Chinnadurai G: Genetic identification of adenovirus type 5 genes that influence viral spread. J Virol 80: 2000-2012, 2006.

31. White E: Mechanisms of apoptosis regulation by viral oncogenes in infection and tumorigenesis. Cell Death Differ 13: 1371-1377, 2006.

32. Cross JR, Postigo A, Blight K and Downward J: Viral prosurvival proteins block separate stages in Bax activation but changes in mitochondrial ultrastructure still occur. Cell Death Differ 15: 997-1008, 2008.

33. Yoon AR, Kim JH, Lee YS, et al: Markedly enhanced cytolysis by E1B-19kD-deleted oncolytic adenovirus in combination with cisplatin. Hum Gene Ther 17: 379-390, 2006.

34. Lomonosova E, Subramanian T and Chinnadurai G: Mitochondrial localization of p53 during adenovirus infection and regulation of its activity by E1B-19K. Oncogene 24: 67966808,2005

35. Matsushita T, Okada T, Inaba T, Mizukami H, Ozawa $\mathrm{K}$ and Colosi P: The adenovirus E1A and E1B19K genes provide a helper function for transfection-based adeno-associated virus vector production. J Gen Virol 85: 2209-2214, 2004.

36. Polster BM, Pevsner J and Hardwick JM: Viral Bcl-2 homologs and their role in virus replication and associated diseases. Biochim Biophys Acta 1644: 211-227, 2004.

37. Tsukuda K, Wiewrodt R, Molnar-Kimber K, Jovanovic VP and Amin KM: An E2F-responsive replication-selective adenovirus targeted to the defective cell cycle in cancer cells: potent antitumoral efficacy but no toxicity to normal cell. Cancer Res 62: 3438-3447, 2002.

38. Rohmer S, Quirin C, Hesse A, et al: Transgene expression by oncolytic adenoviruses is modulated by E1B19K deletion in a cell type-dependent manner. Virology 395: 243-254, 2009. 